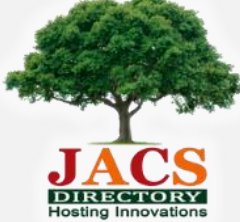

Journal of Nanoscience and Technology

\title{
Synthesis and Characterization of Silver Nanoparticles using Padina pavonica Extract and Evaluation of Their Antibacterial Activity
}

\author{
G. Sudha, A. Balasundaram* \\ PG and Research Department of Zoology, Periyar E.V.R. College (Autonomous), Tiruchirappalli - 620 023, Tamil Nadu, India.
}

\section{ARTICLE DETAILS}

\section{Article history:}

Received 19 May 2018

Accepted 20 June 2018

Available online 07 July 2018

\section{Keywords:}

Padina pavonica

Silver Nanoparticles

Antibacterial Activities

\begin{abstract}
A B S T R A C T
Nanoparticles are the basic essential elements in the wall of nanotechnology and it exhibits fabulous advanced characteristic features based on their properties such as size, morphology and other size dependent properties. In this study, marine algae Padina pavonica (Linn.) was used for the biosynthesis of silver nanoparticles. The biosynthesized silver nanoparticles were characterized with UV-vis spectroscopy, FTIR and SEM. The SEM analysis showed the particle size between $20-70 \mathrm{~nm}$ as well as the polydispersed spherical structure of the nanoparticles. The extract of marine alga P. pavonica (Linn.) as well as silver nanoparticles were tested against gram positive Staphylococcus aureus, Bacillus subtilis and gram negative bacteria as Escherichia coli and Pseudomonas aeruginos. The P. pavonica based silver nanoparticles significantly inhibited the growth of bacteria. The AgNPs possess potential antibacterial activity than algae extract. This novel highly stable spherical polydispersed Ag nanoparticles were synthesized using a simple environmental friendly green method and can be used for antibacterial agent.
\end{abstract}

\section{Introduction}

The enhancing properties of materials in the form of nano sized particles are called nanoparticle. The basic element in the wall of nanotechnology is nanoparticles and it exhibits distinctive features based on their properties such as size, morphology and other size dependent properties [1]. The wide range of application of nanoparticles recorded [2]. Nanoparticles are one of the nearly everyone sought materials for the future significant in many of the fields. Metals like palladium, silver, platinum and gold are exhibit a wide range of materials used for nanoparticles synthesis [3]. A varieties of metal nanoparticles, silver nanoparticles (AgNPs) have received great attention in a diversity of applications, including nanoelectronic devices, sensors, imaging contrast agents, filters, and antimicrobial agents due to their good electrical conductivity, stability, optical property, and antimicrobial activity $[3,4]$. AgNPs widely used for the treatment of cancer. Several in vitro studies using AgNPs have demonstrated their potential as effective anticancer agents [5-8]. In biological method using bacteria, fungi, algae, enzymes and plant extracts used for the synthesis of silver nanoparticles due to their environment benign process and ability of large scale production over physical and chemical methods [9]. Green synthesis of silver nanoparticles by algae extract shows more advantageous over other biological processes are bacteria and fungi, because it eliminates the cell culture maintaining process, and also it more suitable for large scale production of silver nanoparticles [10]. In the present study, the chosen marine brown seaweed is Padina pavonica. The brown algae Padina pavonica (L.) Gaill is wide spread in the Adriatic Sea. It belongs to the family Dictyotaceae, order Dictyotales, subclass Isogeneratae, class Phaeophyceae. In this study, the synthesis of pure and stable metallic nanoparticles of silver by the reduction of aqueous $\mathrm{Ag}^{+}$ions with the broth of marine algae $P$. pavonica and further investigated the impact of the synthesized nanoparticles against antibacterial activity using agar disc diffusion method.

\section{Experimental Methods}

\subsection{Preparation of Seaweed Extract}

The seaweed was dried and made into fine powder. $1 \mathrm{~g}$ of biomass was kept in a $250 \mathrm{~mL}$ conical flask with $100 \mathrm{~mL}$ of Milli Q water for $24 \mathrm{~h}$.
Whatman No. 1 filter paper used to filter the extract and kept in a refrigerator for further studies.

\subsection{Synthesis of Ag Nanoparticles using Seweed Extract}

For the Ag nanoparticle synthesis, $10 \mathrm{~mL}$ seaweed filtrate was added with $90 \mathrm{~mL}$ of $10^{-3} \mathrm{M}$ aqueous $\mathrm{AgNO}_{3}$ solutions at room temperature [11] and kept aside for few hours. The steady formation of silver nanoparticles was confirmed when the solution changed its colour.

\subsection{UV Spectroscopic Analysis}

The silver nanoparticles were examined under visible and UV light. For UV spectrophotometer analysis, the nanoparticles were dispersed in deionized water. Then the sample is diluted to 1:10 with the deionized water. The nanoparticles solution was scanned in the wavelength ranging from 340-900 nm using UV-vis spectrophotometer. The peak values of the UV Spectra were recorded for discussion.

\subsection{Fourier Transform Infrared (FTIR) Spectroscopic Analysis}

The presences of bioactive functional groups are responsible for the reduction of silver ions which were recorded by Perkin Elmer Spectrophotometer system, USA. A small amount of liquid of silver nanoparticle was respectively placed directly on sample holder of the infrared spectrometer with constant pressure applied and data of infrared absorbance collected over the wave number ranged from $4000 \mathrm{~cm}^{-1}$ to 400 $\mathrm{cm}^{-1}$ and computerized for analyses by using the 21 CFR part 11 software.

\subsection{SEM Analysis of Silver Nanoparticles}

Scanning electron microscopic (SEM) analysis carried out by VEGA3 LMU machine. The samples were prepared on a carbon coated copper grid by just dropping a very small amount of the silver nanoparticles on the grid. Extra solution was removed using a blotting paper and then the films on the SEM grid were allowed to dry by putting it under a mercury lamp for $5 \mathrm{~min}$ and then performed for characterization.

\subsection{Microorganisms}

The microbial strains employed in the biological assays were Gram Positive bacteria: Streptococcus aureus (MTCC 96), Bacillus subtilis (MTCC 441), Gram - negative bacteria: Pseudomonas aeruginosa (MTCC2474), Escherichia coli (MTCC 119) which were obtained from microbial type culture collection (MTCC) at the institute of Microbial Technology (IMTECH), Chandigarh, India. 


\subsection{Solutions Preparation for Antimicrobial Experiment}

The dried extract of Padina pavonica was weighed $(10 \mathrm{mg} / \mathrm{mL})$ and dissolved in deionized water. Solutions $1 \mathrm{mM}$ of $\mathrm{AgNO}_{3}$, collected AgNPs $(10 \mathrm{mg} / \mathrm{mL})$, standard solution of chloramphenicol $(25 \mathrm{mg} / \mathrm{mL}$ deionized water) were used in this study.

\subsection{Antimicrobial Assay}

Antibiogram was done by disc diffusion method using plant extracts. Petri plates were prepared by pouring $30 \mathrm{~mL}$ of nutrient agar medium for bacteria/fungi. The test organism was inoculated on solidified agar plate with the help of micropipette and spread and allowed to dry for $10 \mathrm{~min}$. The media was inoculated with bacteria from a broth culture. A sterile cotton swab is dipped into a standardized bacterial/ fungi test suspension and used to evenly inoculate the entire surface of the nutrient agar/PDA plate. Briefly, inoculums containing Streptococcus aureus, Bacillus subtilis, Pseudomonas aeruginosa and Escherichia coli on nutrient agar plates for bacteria. Using sterile forceps, the sterile filter papers ( $6 \mathrm{~mm}$ diameter) containing each $30 \mu \mathrm{L}$ of plant extract and $\mathrm{AgNO}_{3}$ solutions were used. AgNPs and standard solution as chloramphenicol were laid down on the surface of inoculated agar plate. The plates were incubated at $37{ }^{\circ} \mathrm{C}$ for 24

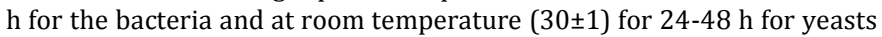
strains. Each samples were tested in triplicate. The antimicrobial potential of test compounds were determined on the basis of mean diameter of zone of inhibition around the disc in millimeters. The zones of inhibition of the tested microorganisms by the extracts were measured using a millimeter scale. The diameter sizes in mm of the zone of inhibition are shown in the Table 1.

\section{Results and Discussion}

The green synthesis of silver nanoparticles through plant extracts were carried out. Silver nitrate is used for nanoparticle synthesis as silver has distinctive properties such as good conductivity, catalytic and chemical stability. Applications of such eco-friendly nanoparticles in bactericidal, wound healing and other medical and electronic applications, makes this method potentially exciting for the large-scale synthesis of other inorganic materials (nanomaterials). The aqueous silver ions when exposed to seaweed extract were reduced in solution, there by leading to the formation of silver hydrosol. The time duration of change in colour varies from algae to algae. The phytochemicals present in the extract were considered as responsible for the reduction of silver ions. It is well known that silver nanoparticles exhibit brown colour in aqueous solution due to excitation of surface plasmon vibrations in silver nanoparticles. The appearance of brown color in Padina pavonica extract treated flask reveals clear indication for the formation of $\mathrm{Ag}$ nanoparticles [12]. Present findings are in good agreement with earlier findings [13].

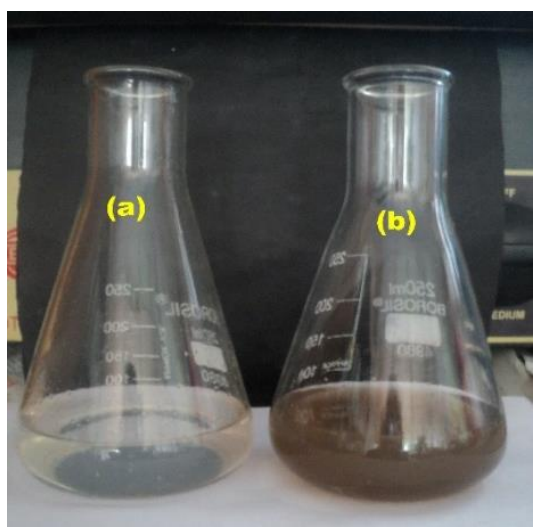

Fig. 1 (a) Aqueous solution of $1 \mathrm{mM} \mathrm{AgNO}_{3}$ before adding the Padina pavonica extract and (b) after addition of Padina pavonica extract at $4 \mathrm{~h}$

The nanoparticles were primarily characterized by UV-visible spectroscopy which provide very useful technique for the analysis of nanoparticles [14]. Fig. 2 shows the UV-vis spectra of silver nanoparticles which were prepared using silver nitrate and Padina pavonica broth. It is observed that the maximum absorbance of Ag nanoparticles occurs at 422 $\mathrm{nm}$. Appearance of this peak, assigned to a surface plasmon which is welldocumented for various metal nanoparticles with size ranging from $2 \mathrm{~nm}$ to $100 \mathrm{~nm}$ [15]. It is observed from the spectra that the surface plasmon resonance band of AgNP's occurs at $422 \mathrm{~nm}$ which is a characteristic peak of AgNP's as reported earlier [12, 16].

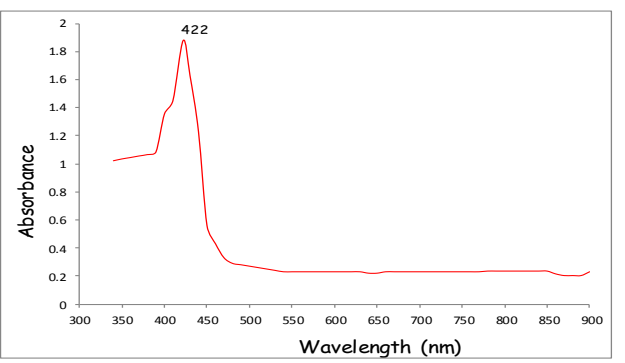

Fig. 2 UV-vis spectrum of silver nanoparticle

Major FTIR peaks were observed at 3,434, 1,630 and $670 \mathrm{~cm}^{-1}$ indicating the possible interaction of biomolecules for capping, and stabilization of Ag nanoparticles (Fig. 3). The shift in peak clearly attributes the reduction of silver ions into their respective nanoparticles. A strong absorption peak at $3,434 \mathrm{~cm}^{-1}$ indicates the presence of phenols and alcohols with free $0-\mathrm{H}$ group. The peak at $1,630 \mathrm{~cm}^{-1}$ represents the presence of amide I group and may well arise due to carbonyl stretch in organic molecules [17]. The peak at $670 \mathrm{~cm}^{-1}$ may be assigned due to the stretching vibration of aromatic ring that might be attached to the free 0 $\mathrm{H}$ group. Satyavani et al. [18] have reported that silver ions may possibly bind to phenolic compounds with one or more aromatic ring resulting in the formation of Ag nanoparticles.

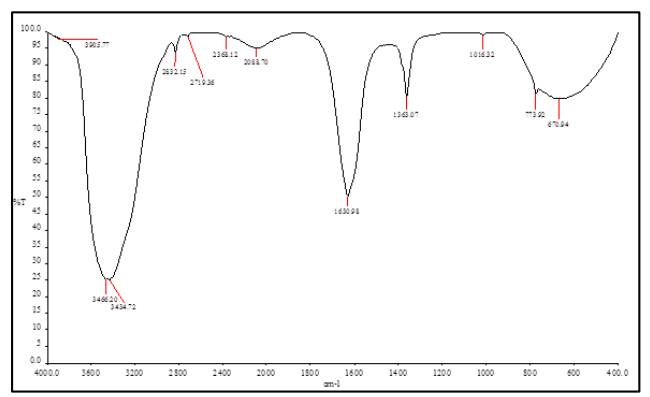

Fig. 3 FTIR Spectroscopic analysis of silver nanoparticle

SEM analysis was carried out to understand the topology and the size of the Ag-NPs. The SEM image (Fig. 4) shows the high density silver nanoparticles synthesized by the seed extract with the development of silver nanostructures. Most of the nanoparticles aggregated and only a few of them were scattered as observed under SEM. The SEM analysis reveals the particle size between $20-70 \mathrm{~nm}$ as well the spherical structure of the nanoparticles which looks similar with earlier reports [13].

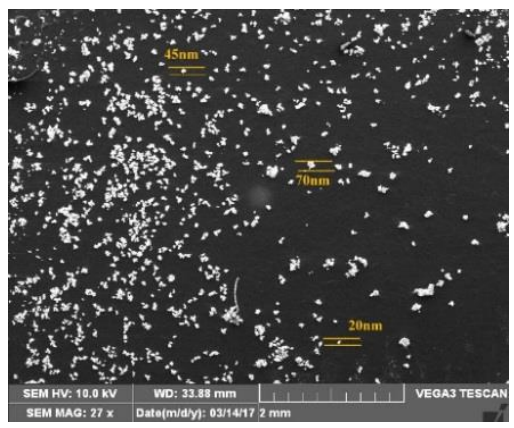

Fig. 4 High resolution scanning electron microscopic (SEM) images of silver nanoparticles (AgNPs)

Toxicity studies on pathogen opens a door for nanotechnology applications in medicine. Biological synthesis of metal NPs is a traditional method and the use of plant extract has a new awareness for the control of disease, besides being safe and no phytotoxic effects. The biologically synthesized silver nanoparticles using medicinal plants were found to be highly toxic against different pathogenic bacteria of selected species. The SNPs of Padina pavonica shows highest antibacterial activity was observed against Streptococcus aureus, Bacillus subtilis, Pseudomonas aeruginosa and Escherichia coli. The inhibitory activities in culture media of the Ag nanoparticles reported in Table 1 were comparable with standard antimicrobiotic viz. chloramphenicol.

E. coli and $S$. auerus, which already known to be multi-resistant to antibiotics, were resistant to tested AgNPs, Padina pavonica extract and $\mathrm{AgNO}_{3}$. The mean inhibition zone of $\mathrm{AgNO}_{3}$, Padina pavonica extract and AgNPs were $2.10 \pm 0.03,3.20 \pm 0.10$ and $7.50 \pm 0.09 \mathrm{~mm}$ respectively for E. coli. The mean inhibition zone of $\mathrm{AgNO}_{3}$, Padina pavonica extract and 
AgNPs were $2.50 \pm 0.02,4.30 \pm 0.04$ and $8.60 \pm 0.09 \mathrm{~mm}$ respectively for S. auerus. The mean inhibition zone for standard is $10.40 \pm 0.04$ and 11.30 \pm 0.09 for $E$. coli and $S$. auerus respectively.

Bacillus subtilis and Pseudomonas aeruginosa, are known to be multiresistant to antibiotics, were resistant to tested $\mathrm{AgNO}_{3}$, Padina pavonica extract and AgNPs. The mean inhibition zone of AgNPs, Padina pavonica extract and $\mathrm{AgNO}_{3}$ were $2.40 \pm 0.03,3.30 \pm 0.05$ and $9.40 \pm 0.06 \mathrm{~mm}$ respectively for Bacillus subtilis. The mean inhibition zone of $\mathrm{AgNO}_{3}$, Padina pavonica extract and AgNPs were $2.50 \pm 0.04,1.80 \pm 0.02$ and 8.30 $\pm 0.09 \mathrm{~mm}$ respectively for Pseudomonas aeruginosa. The mean inhibition zone for standard is $10.30 \pm 0.05$ and $9.80 \pm 0.07$ for Bacillus subtilis and Pseudomonas aeruginosa respectively.

The results showed that the antimicrobial activity was directly proportional to the concentration of AgNPs, Padina pavonica extract, $\mathrm{AgNO}_{3}$ and Standard. The AgNPs shows highest antimicrobial activity which was observed against Streptococcus aureus, Bacillus subtilis, Pseudomonas aeruginosa and Escherichia coli. The AgNPs possess similar activity to standard drug as chloramphenicol for bacteria.

Table 1 Antibacterial activity of $\mathrm{AgNO}_{3}$, Padina pavonica extract and AgNPs

\begin{tabular}{lcllll}
\hline Samples & $\begin{array}{l}\text { Conc. } \\
(\mu \mathrm{L})\end{array}$ & $\begin{array}{l}\text { E. coli } \\
(\mathrm{mm})\end{array}$ & $\begin{array}{l}\text { S. auerus } \\
(\mathrm{mm})\end{array}$ & $\begin{array}{l}P . \\
\text { aeruginosa } \\
(\mathrm{mm})\end{array}$ & $\begin{array}{l}\text { B. subtilis } \\
(\mathrm{mm})\end{array}$ \\
\hline $\mathrm{AgNO}_{3}$ & 30 & $2.10 \pm 0.03$ & $2.50 \pm 0.02$ & $2.50 \pm 0.04$ & $2.40 \pm 0.03$ \\
$\begin{array}{l}\text { Padina pavonica } \\
\text { extract }\end{array}$ & 30 & $3.20 \pm 0.10$ & $4.30 \pm 0.04$ & $1.80 \pm 0.02$ & $3.30 \pm 0.05$ \\
AgNPs & 30 & $7.50 \pm 0.09$ & $8.60 \pm 0.09$ & $8.30 \pm 0.09$ & $9.40 \pm 0.06$ \\
Standard & 30 & $10.40 \pm 0.04$ & $11.30 \pm 0.09$ & $9.80 \pm 0.07$ & $10.30 \pm 0.05$ \\
\hline
\end{tabular}

Mean $\pm S D$; Standard = Chloramphenicol
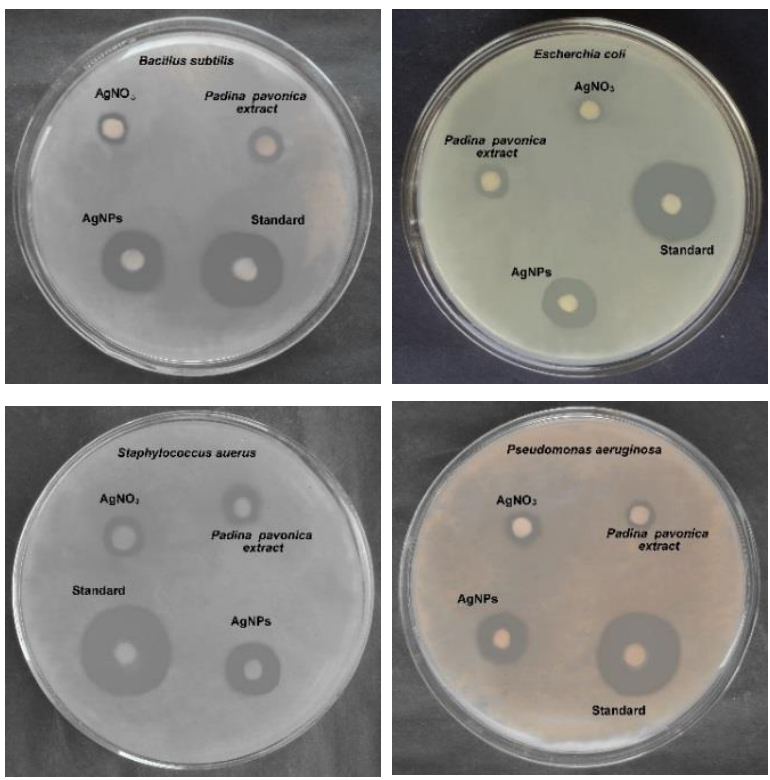

Fig. 5 Antibacterial activity of $\mathrm{AgNPs}, \mathrm{AgNO}_{3}$ and Padina pavonica extract

In this study, to evaluate the antibacterial effects of Ag nanoparticles against various microorganisms, we used four representative microorganisms, Streptococcus aureus, Bacillus subtilis, Pseudomonas aeruginosa and Escherichia coli. When Ag nanoparticles were tested, they effectively inhibited bacterial growth. In our results, Ag nanoparticles showed antimicrobial activity against E. coli (Fig. 5) that was similar to that found by Sondi et al. [19]. In contrast, the inhibitory effect of Ag nanoparticles was mild in S. aureus and B. subtilis (Fig. 5) as compared with other microorganisms; these results suggest that the antimicrobial effects of $\mathrm{Ag}$ nanoparticles may be associated with characteristics of certain bacterial species. The growth of microbes were inhibited by AgNPs showed a difference in the inhibition of growth of microbes due to the presence of peptidoglycan. Peptidoglycan is a complex structure have a strong negative charges contains lipoteichoic acids or teichoic acids. This charge could provide to the sequestrated of free Ag+. Thus gram negative bacteria may permit more silver to reach the cytoplasmic membrane than the gram positive bacteria [20]. The lesser ability of the Ag nanoparticles against $S$. aureus and $B$. subtilis may derive from the membrane structure. The specific membrane feature of peptidoglycan present only in bacterial cells. Therefore, antibacterial activity of silver nanoparticles is related with the peptidoglycan layer, it would be easier and more precise to use silver nanoparticles as an antibacterial agent. The AgNPs synthesized from algae species are efficient to multi-drug resistant microbes.

\section{Conclusion}

The present study conclude that the bio-reduction of silver ions using Padina pavonica extract achieved by the exposure of aqueous silver ions with the extract and hence the synthesis of silver nanoparticles were confirmed by the change of colour of the extract mixture. These environmentally benign silver nanoparticles were further confirmed by using SEM. The SEM analysis showed the particle size between $20-70 \mathrm{~nm}$ as well the spherical structure of the nanoparticles. The results indicated that silver nanoparticles have good antibacterial activity against different microorganisms such as Streptococcus aureus, Bacillus subtilis, Pseudomonas aeruginosa and Escherichia coli. It is confirmed that silver nanoparticles are capable of rendering high antibacterial efficacy and hence has a great potential in the preparation of drugs used against bacterial diseases. Applications of $\mathrm{Ag}$ nanoparticles based on these findings may lead to valuable discoveries in various fields such as medical devices and antimicrobial systems.

\section{References}

[1] A.M. Smith, H. Duan, M.N. Rhyner, G. Ruan, S.A. Nie, A systematic examination of surface coatings on the optical and chemical properties of semiconductor quantum dots, Phys. Chem. Chem. Phys. 8 (2006) 3895-3903.

[2] M. Rai, A. Yadav, A. Gade, Silver nanoparticles as a new generation of antimicrobials, Biotechnol. Adv. 27 (2009) 76-83.

[3] P. Rauwel, E. Rauwel, S. Ferdov, M.P. Singh, Silver nanoparticles: Synthesis, properties, and applications, Adv. Colloid Interf. Sci. 6 (2015) 624394-624408.

[4] C. Krishnaraj, E. Jagan, S. Rajasekar, P. Selvakumar, P. Kalaichelvan, N. Mohan, Synthesis of silver nanoparticles using Acalypha indica leaf extracts and its antibacterial activity against water borne pathogens, Colloids Surf. B 76 (2010) 50-56.

[5] P.V. Asha Rani, G. Low Kah Mun, M.P. Hande, S. Valiyaveettil, Cytotoxicity and genotoxicity of silver nanoparticles in human cells, ACS Nano. 3 (2009) 279290.

[6] K. Vasanth, K. Ilango, R. Mohan Kumar, A. Agrawal, G.P. Dubey, Anticancer activity of Moringa oleifera mediated silver nanoparticles on human cervical carcinoma cells by apoptosis induction, Colloids Surf. B 117 (2014) 354-359.

[7] R. Gengan, K. Anand, A. Phulukdaree, A. Chuturgoon, A549 lung cell line activity of biosynthesized silver nanoparticles using Albizia adianthifolia leaf, Colloids Surf. B 105 (2013) 87-91.

[8] M.A. Farah, M.A. Ali, S.M. Chen, Y. Li, F.M. Al-Hemaid, F.M. Abou-Tarboush, et al., Silver nanoparticles synthesized from Adenium obesum leaf extract induced DNA damage, apoptosis and autophagy via generation of reactive oxygen species, Colloids Surf. B 141 (2016) 158-169.

[9] M. Gnanadesigan, M. Anand, S. Ravikumar, M. Maruthupandy, M. Syed Ali, et al., Antibacterial potential of biosynthesised silver nanoparticles using Avicennia marina mangrove plant, Appl. Nanosci. 2(2) (2012) 143-147.

[10] M. Singh, R. Kalaivani, S. Manikandan, N. Sangeetha, A.K. Kumaraguru, Facile green synthesis of variable metallic gold nanoparticle using Padina gymnospora, a brown marine macroalga, Appl. Nanosci. 17 (2012) 145-151.

[11] K. Govindaraju, V. Kiruthiga, V. Ganesh Kumar, G. Singaravelu, Extracellular synthesis of silver nanoparticles by a marine alga, Sargassum wightii Grevilli and Their Antibacterial Effects, J. Nanosci. Nanotechnol. 9 (2009) 5497-5501.

[12] A. Ahmad, P. Mukherjee, S. Senapati, D. Mandal, M.I. Khan, R. Kumar, M. Sastry, Extracellular biosynthesis of silver nanoparticles using the fungus Fusarium oxysporum, Colloids Surf. B 28 (2003) 313-318.

[13] K. Sahayaraj, S. Rajesh, J.M. Rathi, Silver nanoparticles biosynthesis using marine alga Padina pavonica (Linn.) and its microbicidal activity, Dig. J. Nanomater. Bios. 7(4) (2012) 1557-1567.

[14] M. Sastry, V. Patil, S.R. Sainkar, Electrostatically controlled diffusion of carboxylic acid derivatized silver colloidal particles in thermally evaporated fatty amine films, Jour. Phys. Chem. B 102 (1998) 1404-1409.

[15] A. Henglein, Physicochemical properties of small metal particles in solution: "Microelectrode" reactions, chemisorption, composite metal particles, and the atom-to-metal transition, J. Phys. Chem. 97 (1993) 5457-5471.

[16] G. Singaravelu, J. Arockiamary, K. Ganesh, K. Govindaraju, A novel extracellular synthesis of monodisperse gold nanoparticles using marine alga, Sargassum wightii Greville, Colloids Surf. B. Biointerf. 57 (2007) 97-101.

[17] P. Kumar, S. Senthamilselvi, A. Lakshmipraba, K. Premkumar, R. Muthukumaran, et al., Efficacy of biosynthesized silver nanoparticles using Acanthophora spicifera to encumber biofilm formation, Dig. J. Nanomater. Bios. 7 (2012) 511-522.

[18] K. Satyavani, S. Gurudeeban, T. Ramanathan, T. Balasubramanian, Biomedical potential of silver nanoparticles synthesized from calli cells of Citrullus colocynthis (L.), J. Nanobiotechnol. 9 (2011) 43-50.

[19] I. Sondi, A. Salopek-Sondi, Silver nanoparticles as antimicrobial agent: a case study on E. coli as a model for Gram-negative bacteria, J. Colloid. Interf. Sci. 275 (2004) 177-182.

[20] N. Ahmad, S. Sharma, V.N. Singh, S.F. Shamsi, A. Fatma, B.R. Mehta, Biosynthesis of silver nanoparticles from Desmodium trifolium: A novel approach towards weed utilization, Biotechnol. Res. Int. 1 (2011) 8-12. 